\title{
Comparison of SC-FDMA and HSUPA in the Return- Link of Evolved S-UMTS Architecture
}

\author{
S.Janaaththanan, C. Kasparis, and Barry G. Evans, \\ Centre for Communication Systems Research, \\ University of Surrey, \\ Guildford, GU2 7XH, United Kingdom. \\ \{S.Janaaththanan, C.Kasparis,B.Evans\} @ surrey.ac.uk
}

\begin{abstract}
Single Carrier Frequency Division Multiple Access (SC-FDMA), a multiple access technique similar to Orthogonal Frequency Division Multiple Access (OFDMA), presently features as a strong candidate in the return link of the Long Time Evolution (LTE) of T-UMTS. In the satellite domain there is an emerging research interest in extending mobile broadcasting system architectures that include complementary ground components, in providing bi-directional data services to mobile users. In such 'evolved S-UMTS' architecture, compatibility with current (CDMA based) and future (OFDMA/SC-FDMA) based) T-UMTS physical layer techniques is of high relevance, if satellite networks are to assume a complementary role to terrestrial ones. In this paper, we first give an overview of SC-FDMA and then compare, via simulations, the performance of the technique with that of the WCDMA-based High Speed Uplink Packet Access (HSUPA) that has been defined in Release 6 of T-UMTS. The performance evaluations are performed over channels that are representative of the evolved S-UMTS architectures. Emphasis in the study is also given to realistic channel estimation and taking into account the effects of the amplifier's non-linearity. Other elements addressed in this paper are the impact of linearization techniques, and the pilot channel design in order to achieve robust estimation in the presence of non-linear channels.
\end{abstract}

Keywords-SC-FDMA, HSUPA, LTE, DFT, TWTA.

\section{INTRODUCTION}

The delivery of broadcasting services to handheld devices via hybrid satellite-terrestrial networks is already a commercial reality in the far-east and is also expected to be available in Europe in the near future [1]. The efficiency of such networks in supporting high-speed links even in urban environments is an important motivation in considering an upgrade of the hybrid system in supporting bi-directional data services. Among other issues, such extension would require to choose a physical layer/multiple access technology. A crucial factor in making this choice is compatibility with T-UMTS, where presently CDMA is adopted, and SC-FDMA is considered as a strong 'LTE' candidate for achieving better spectral efficiency and robustness with respect to amplifier non-linearity, relative to OFDMA [2].

SC-FDMA [2], also called Discrete Fourier Transform (DFT) spread Orthogonal Frequency Division Multiple Access (OFDMA), performs the DFT spreading, in the transmitter, prior to the Inverse Discrete Fourier Transform (IDFT) block. With this DFT block, it spreads the energy of individual modulation symbols over a number of sub-carriers in the DFT block. Figure 1 shows the block diagram of the signal processing blocks involved in SC-FDMA. It is observed that the main difference between OFDMA and SC-FDMA is the DFT and inverse DFT (IDFT) blocks at the transmitter and receiver, respectively. Depending on the ratio between the number of DFT sub-carriers and the number of IDFT subcarriers at the transmitter, the DFT spreading cancels the mixing of signals caused by the IFFT, and thus generates transmitted signals with lower Peak-to-Average Power Ratio (PAPR) than traditional OFDMA signals [3],[4].

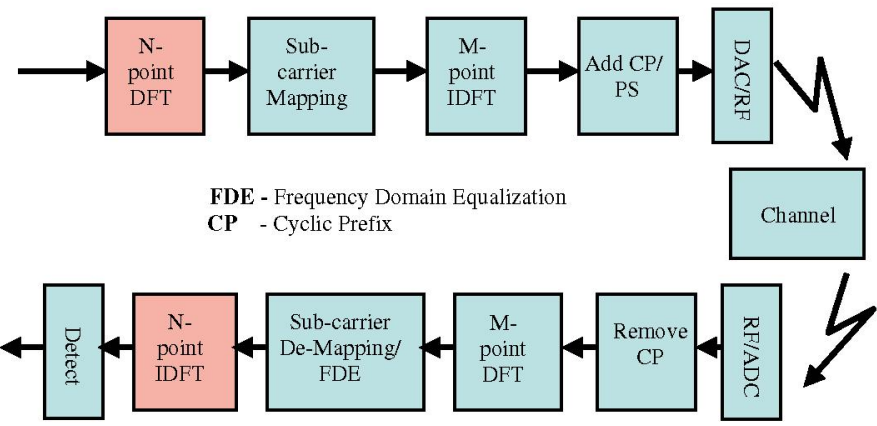

Fig. 1. Signal Processing Block Diagram of SC-FDMA Scheme [4].

SC-FDMA is classified into two types depending on how the DFT spread symbols are mapped onto the sub-carriers in the IDFT block- Localized SC-FDMA (L-SC-FDMA) and Interleaved SC-FDMA (I-SC-FDMA). As shown in Figure 2, in L- SC-FDMA the DFT spread symbols are mapped onto consecutive points in the IDFT block, whereas in I-SCFDMA, the symbols are mapped onto sub-carriers which are a certain distance apart. Thus, in the fading channel, I-SCFDMA exploits the frequency diversity more efficiently. At the same time, it has lower PAPR when compared with the Localized SC-FDMA. However, interleaved mapping is more challenging for channel estimation, and extracting synchronization parameters. Here we focus our study mainly on L-SC-FDMA, as it is under investigation for 3GPP LTE [3], [4], [5]. 

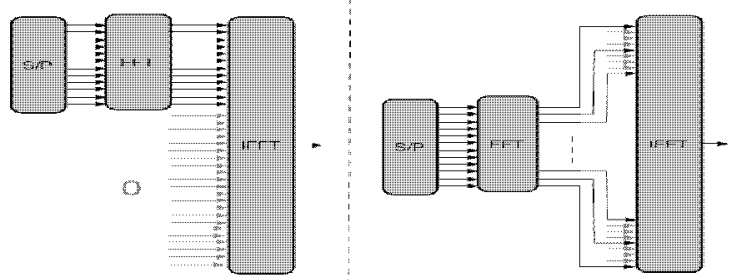

Fig. 2. Sub-carrier mapping of Localized and Interleaved SC-FDMA

In this study we also consider the effect ofa pre-distortion technique for mitigating the effects of the non-linear Traveling Wave Tube Amplifier (TWTA).

The remainder of this paper is organized as follows: in Section II, we introduce the system modeling of SC-FDMA, HSUPA, Power Amplifier, and Polynomial based Predistorters. Section III provides the simulation parameters and the frame structures used. In Section IV, we report the comparative results between SC-FDMA and HSUPA systems under different mobile satellite channel scenarios. Furthermore, the investigation of a CAZAC (Constant Amplitude Zero Autocorrelation) pilot sequence in the nonlinear channel estimation for SC-FDMA is also included. Finally, Section IV concludes the paper.

\section{SYSTEM MODELLING}

In this Section, a brief overview of SC-FDMA is provided with the help of matrix notations [6]. Thereafter, the performance characteristics of the TWTA and the modeling of Polynomial Pre-distorter are given.

\section{A. SC-FDMA}

The discrete base-band model of a standard SC-FDMA system was incorporated for analysis, as shown in Figure 1. Let $\mathbf{u}_{\mathbf{i}}$ be the $i^{\text {th }}$ block of transmitted data, which consists of $L-$ QPSK symbols. The first processing stage is DFT spreading, denoted by the linear DFT matrix $\mathbf{Q}$ of dimension $M \times M$. Then, the zero insertion and sub-carrier mappings are modeled as linear transformations on the DFT spread signal, represented by the matrix $\mathbf{M}$. Remaining baseband stages at the transmitter are the IFFT and CP insertion, which are modeled by the linear matrices $\mathbf{F}^{-1}$ and $\mathbf{T}_{\mathbf{C P}}$, respectively. Thus, following all these processing stages the transmit SC-FDMA signal is given as:

$$
\mathbf{x}_{\mathrm{i}}=\mathbf{T}_{\mathbf{C P}} \mathbf{F}^{-\mathbf{1}} \mathbf{M Q \mathbf { u } _ { \mathrm { i } }}
$$

Provided that timing is recovered accurately at the receiver, the received signal is given as:

$$
\mathbf{y}_{\mathbf{i}}=\mathbf{H}_{\mathbf{0}} \mathbf{x}_{\mathbf{i}}+\mathbf{H}_{1} \mathbf{x}_{\mathbf{i}-1}+\mathbf{n}
$$

where $\mathbf{n}$ is a vector which contains samples of the Gaussian noise (zero mean and variances of $\sigma^{2}$ ). In (1), it is assumed that the channel is modeled as an FIR filter of $L+1$ taps; $\mathbf{h}=[h(0), \ldots h(L)]$, and $\mathbf{H}_{O}$ represents the $P \times P$ upper triangular Toeplitz channel filtering matrix, which is given as:

$$
\mathbf{H}_{O}=\left[\begin{array}{ccccc}
h(0) & \ldots & 0 & 0 & 0 \\
\vdots & h(0) & 0 & 0 & 0 \\
h(L) & \vdots & h(0) & 0 & 0 \\
0 & h(L) & \vdots & \ddots & 0 \\
0 & 0 & h(L) & \ldots & h(0)
\end{array}\right]
$$

$\mathbf{H}_{1}$ models the edge effect caused by the excess delay spread of the propagation channel and results in ISI with previously transmitted symbol $\mathbf{x}_{i-1}$.

$$
\mathbf{H}_{1}=\left[\begin{array}{ccccc}
0 & \ldots & h(L) & \ldots & h(1) \\
\ldots & \ldots & 0 & h(L) & \ldots \\
0 & 0 & 0 & 0 & h(L) \\
\ldots & \ldots & \ldots & \ldots & \ldots \\
0 & 0 & 0 & \ldots & 0
\end{array}\right]
$$

The removal of the $\mathrm{CP}$ at the receiver can be modeled by a linear matrix: $\mathbf{T}_{R}=\left[\mathbf{0}_{K \times C}, \mathbf{I}_{K \times K}\right]$, which effectively also removes the second term in the summation in (1). Thus the resulting signal is given as:

$$
\overline{\mathbf{y}}_{i}=\mathbf{T}_{R} \mathbf{H}_{0} \mathbf{T}_{C P} \mathbf{F}^{-1} \mathbf{M Q \mathbf { u } _ { i }}+\mathbf{T}_{R} \mathbf{n}
$$

The effect of the inclusion and removal of the $\mathrm{CP}$ is that the effective channel matrix $\hat{\mathbf{H}}$ has a circulant structure:

$$
\hat{\mathbf{H}}=\left[\begin{array}{ccccccc}
h(0) & 0 & \ldots & 0 & h(L) & \ldots & h(1) \\
\ldots & h(0) & \ldots & \ldots & 0 & h(L) & \ldots \\
h(L) & \ldots & \ldots & 0 & \ldots & 0 & h(L) \\
0 & h(L) & \ldots & h(0) & 0 & \ldots & 0 \\
\ldots & 0 & \ldots & \ldots & h(0) & 0 & \ldots \\
0 & \ldots & \ldots & h(L) & \ldots & h(0) & 0 \\
0 & 0 & \ldots & 0 & h(L) & \ldots & h(0)
\end{array}\right]
$$

Since, any circulant matrix is diagonalized by the FFT matrix:

$$
\mathbf{\Lambda}=\mathbf{F}_{\mathbf{H}} \mathbf{F}^{-1}
$$

where $\boldsymbol{\Lambda}=\operatorname{diag}\left(\lambda^{0}, \lambda^{1}, \ldots, \lambda^{K-1}\right)$ and $\lambda^{i}$ is the channel response at the $i^{\text {th }}$ frequency bin.

At the receiver, following the FFT processing and channel estimation (using localized pilot tones transmitted in the short blocks), MMSE equalization is performed in the frequency domain (prior to IDFT dispreading). Denoting $\hat{\lambda}_{i}$ as the estimated channel response of the $i^{\text {th }}$ sub-channel, the MMSE estimate of the signal sample on the $i^{\text {th }}$ sub-carrier, is obtained as:

$$
\hat{x}_{i}=\frac{\hat{\lambda}_{i}^{*}}{\hat{\lambda}_{i} \hat{\lambda}_{i}+1 / \mathrm{SNR}} z_{i}
$$

Where $z_{i}$ and $\hat{x}_{i}$ are the received signal at the $i^{\text {th }}$ sub-carrier and its corresponding estimate, respectively. Finally, the equalized symbol is de-spread using the IDFT operation and this followed by demodulation and detection in the time domain. 


\section{B. Travelling Wave Tube Amplifier Characteristics}

Figure 3 describes the non-linear nature of the used TWTA amplifier [1]. These characteristics were used in EU-FP6 project MAESTRO and are based on actual measurements.

\section{Polynomial based-Predistorter}

In order to compensate the non-linear distortion introduced by the TWTA amplifier, a pre-distortion technique is utilized at the transmitter. The polynomial based pre-distorter is considered here, since it converges faster than the Look Up Table (LUT) based pre-distorter [7]. For simplicity, second order polynomial pre-distorter is modeled with the following coefficients;

$$
\begin{aligned}
& y_{-} \text {amp }=\left(0.4503 \mathrm{~A}^{2}-0.0682 \mathrm{~A}+0.5198\right) \\
& y_{-} \text {pha }=\left(-0.0448 \mathrm{~A}^{2}-0.5082 \mathrm{~A}+0.0168\right)
\end{aligned}
$$

Where A denotes the amplitude of the SC-FDMA signal sample. Parametes y_amp and y_pha represent the corresponding amplitude and phase distortion introduced by the Polynomial pre-distorter.
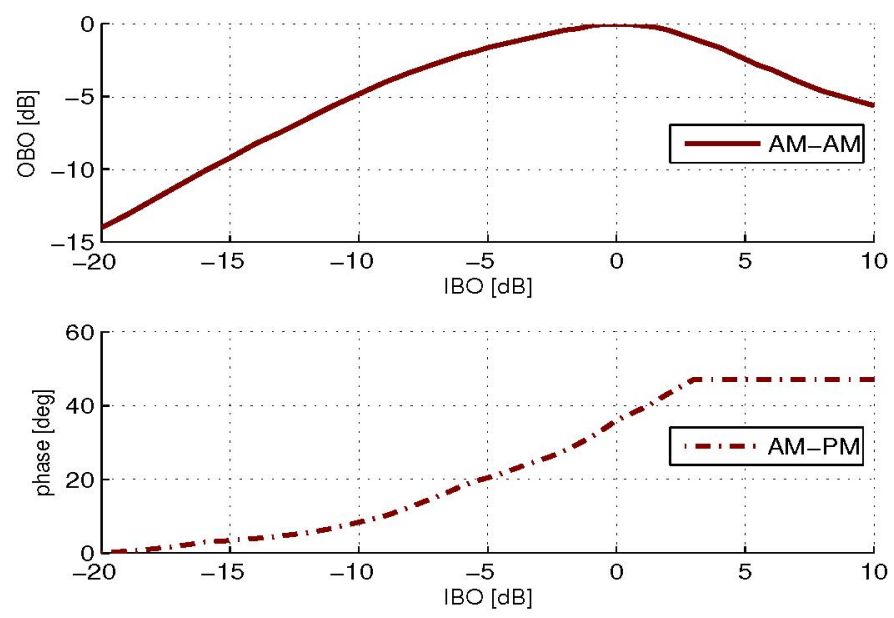

Fig. 3. AM-AM and AM-PM characteristics of a S-band Traveling Wave Tube Amplifier (TWTA) [1].

\section{SIMULATION PARAMETERS}

This section provides the simulation parameters used in this study and the corresponding frame structures used both in SCFDMA and WCDMA (HSUPA). Furthermore, these simulations were performed on three different multi-path channel profiles: a). satellite only with Line of Sight (LOS), b).satellite only with Non-Line of Sight (NLOS), and c). satellite with three terrestrial repeaters (urban environment). Figure 4 shows the normalized power delay profile of these three channels, respectively [1]. In the tapped-delay line model derived from these profiles, taps are assumed to follow rayleigh fading; except for the satellite path which is Rician with a Rice factor of $10 \mathrm{~dB}$. Table 1 and Table 2 list the simulated transmission parameters for WCDMA (HSUPA) and SC-FDMA, respectively. For SC-FDMA, coding and modulation were selected as those of the WCDMA (HSUPA) reference parameter [2], [8].

Considering the simulation of WCDMA (HSUPA), a $10 \mathrm{~ms}$ TTI was considered, although the standard also supports a TTI of $2 \mathrm{~ms}$. In HSDPA, the Enhanced Dedicated Physical Data Channel (E-DPDCH) is defined for carrying data and the Enhanced Dedicated Physical Control Channel (E-DPCC) is defined for the purposes of channel estimation, which is transmitted in parallel to the E-DPDCH. In particular, following spreading, the E-DPCC chips are mapped onto the orthogonal component of the QPSK signal, while E-DPDCH chips are mapped onto the in-phase component. In the simulations, the total transmit power is assumed to be equally divided between the two channels [8].
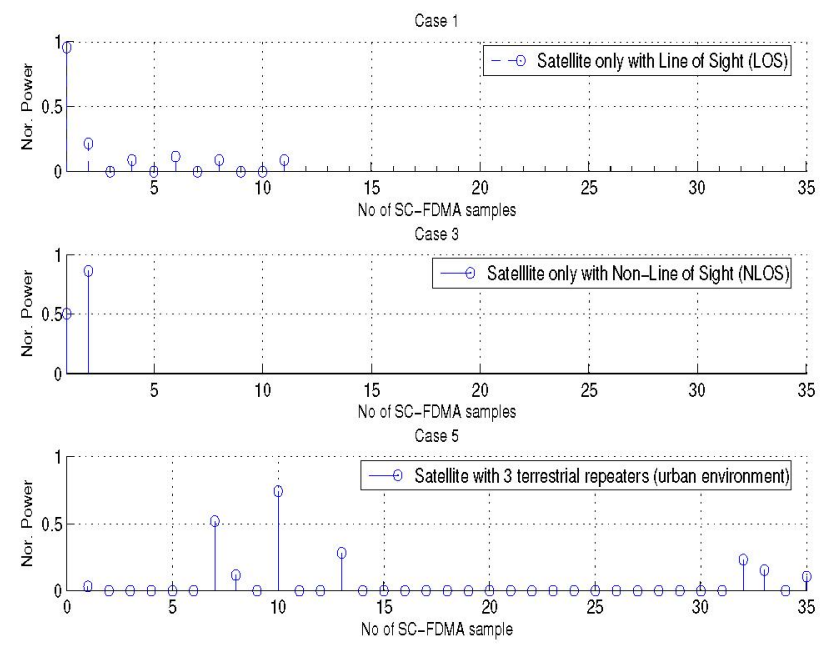

Fig. 4. Multi-path Power Delay profile, representive for evolved S-UMTSSampling frequency $=7.68 \mathrm{MSamples} / \mathrm{Sec}$. [1].

TABLE 1: TRansmission PaRAMETERS USED For the SimUlation OF

\begin{tabular}{|c|c|c|c|c|c|c|c|}
\hline $\begin{array}{c}\text { Transport } \\
\begin{array}{c}\text { Block } \\
\text { Size }\end{array}\end{array}$ & $\begin{array}{c}\text { Number } \\
\text { of Code } \\
\text { Blocks }\end{array}$ & $\begin{array}{c}\text { Modulati } \\
\text { on }\end{array}$ & $\begin{array}{c}\text { OVSF } \\
\text { Code }\end{array}$ & $\begin{array}{c}\text { Code } \\
\text { Rate }\end{array}$ & $\beta_{c}$ & $\beta_{e u}$ & $\begin{array}{c}\text { Rate } \\
\text { after 2 } \\
\text { Tx } \\
(\mathrm{kbps})\end{array}$ \\
\hline 2560 & 1 & QPSK & $\mathrm{C}(4,1)$ & 0.33 & 15 & 30 & 128 \\
\hline
\end{tabular}

For the simulation of SC-FDMA, the sub-frame format and transmission parameters defined in [2] were adopted. The subframe format is shown in figure 2; each sub-frame consists of 6 long blocks obtained from the IFFT operation and with the corresponding $\mathrm{CP}$ insertion, 2 short blocks carrying the reference signal are multiplexed in between the long blocks as shown in Figure 5.

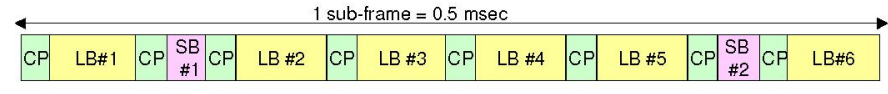

Fig. 5. Sub-Frame Format in SC-FDMA with two Short Blocks per SubFrame [2]. 
TABLE 2: TRANSmission Parameters used For the Simulation of SCFDMA [2]

\begin{tabular}{|c|c|}
\hline Parameter & Value \\
\hline Assumed Spectrum Allocation & $5 \mathrm{MHz}$ \\
\hline Symbol Rate & $7.68 \mathrm{Ms} / \mathrm{s}$ \\
\hline Sub-Frame Duration & $0.5 \mathrm{~ms}$ \\
\hline $\begin{array}{c}\text { Long Block Size ( microsecond/ } \\
\text { Number of occupied sub- } \\
\text { carriers/Number of Samples) }\end{array}$ & $66.67 / 300 / 512$ \\
\hline $\begin{array}{c}\text { Short Block Size ( microsecond/ } \\
\text { Number of occupied sub- } \\
\text { carriers/Number of Samples) }\end{array}$ & $33.33 / 150 / 256$ \\
\hline CP duration & $\begin{array}{c}(4.04 / 31) \times 7 \text { (i.e. for the 7 out of } 8 \\
\text { blocks in the sub-frame) } \\
(5.08 / 39) \times 1 \text { (i.e. for the } 1 \text { out of } 8 \\
\text { blocks in the sub-frame) }\end{array}$ \\
\hline $\begin{array}{c}\text { Sub-Carriers per Resource Unit } \\
25 \text { (6 Resource Units per Long } \\
\text { Block) }\end{array}$ \\
\hline Resource Unit Mapping Type & Localized \\
\hline Points in the DFT Spreading & 25 \\
\hline $\begin{array}{c}\text { Reference Signal Structure for } \\
\text { Uplink Channel Estimation }\end{array}$ & $\begin{array}{c}\text { Localized sub-carriers (carried by the } \\
\text { short-blocks) }\end{array}$ \\
\hline
\end{tabular}

\section{SIMULATION RESULTS}

This section presents the simulation results used for comparing and evaluating the performance, based on the BLER Vs Eb/No curves, of SC-FDMA and WCDMA (HSUPA) in the return link of the evolved S-UMTS. In all cases, a vehicular speed of $100 \mathrm{~km} / \mathrm{ph}$ was assumed.

Figure 6 gives the simulation results obtained for three different multi-path channels, assumed perfect channel estimation. In this case, complete transmit power is allocated to the data channel and no power allocation to the pilot channel. It is observed that both schemes provide approximately the same performance over channel cases 1 \& 3. However, WCDMA performs better over the channel 5, which is a combined 'satellite+repeater' channel. This is due to the rake receiver model adopted with the WCDMA system, which (has six fingers) is able to detect long delayed channel taps by allocating fingers to them. On the other hand, the SCFDMA receiver's performance is mainly limited by the cyclic prefix length, which is significantly smaller than the delay spread of the channel-5.

Figure 7 gives similar results as in Figure 6, but with realistic (pilot based) channel estimation. As explained in the previous section, in HSUPA $50 \%$ of the available power is allocated to the E-DCPCC (recommended in the HSUPA standard). This will, in turn, result in $3 \mathrm{~dB}$ loss in the performance. On the other hand, in SC-FDMA, 26 pilot tones (and 150 data symbols) are transmitted per sub-frame $(\sim 1 / 7)$, and the power allocation (between data and pilots) was also kept at this proportion. Therefore, the channel estimates in WCDMA are more reliable than that of SC-FDMA due to the fact that more power is allocated to pilots in WCDMA and together with the presence of noise averaging effects in channel estimation, which is not present in SC-FDMA. However, the overall performance of the two systems is similar to Figure 6.

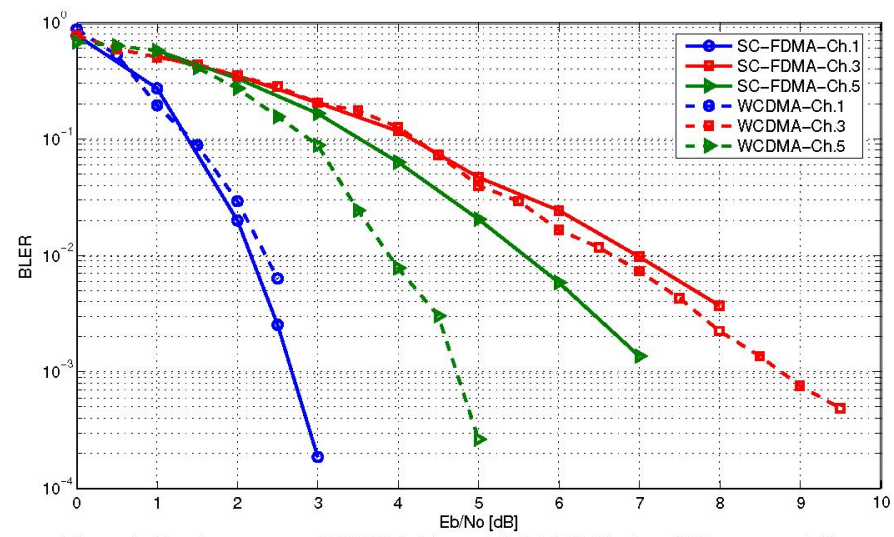

Fig. 6. Performance of WCDMA and SC-FDMA for different mobile channel profiles with perfect channel estimation.

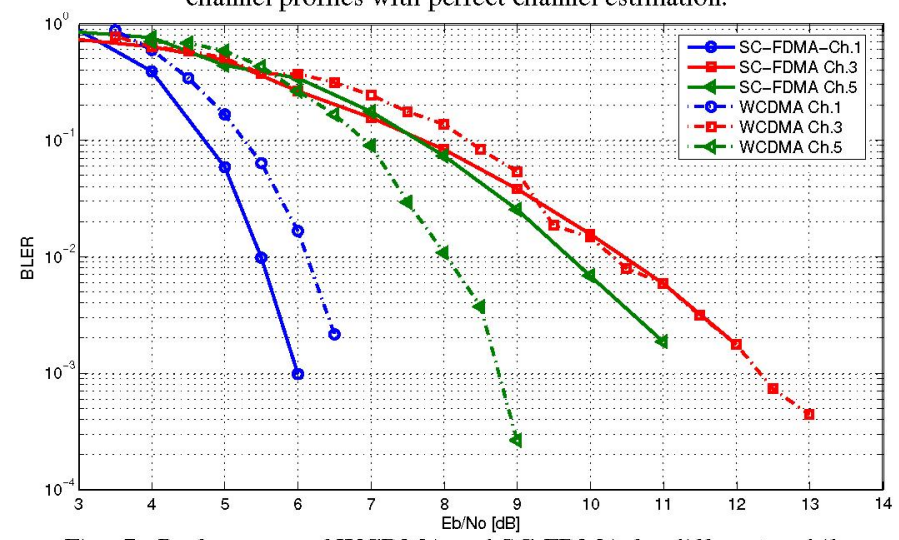

Fig, 7. Performance of WCDMA and SC-FDMA for different mobile channel profiles with realistic channel estimation.

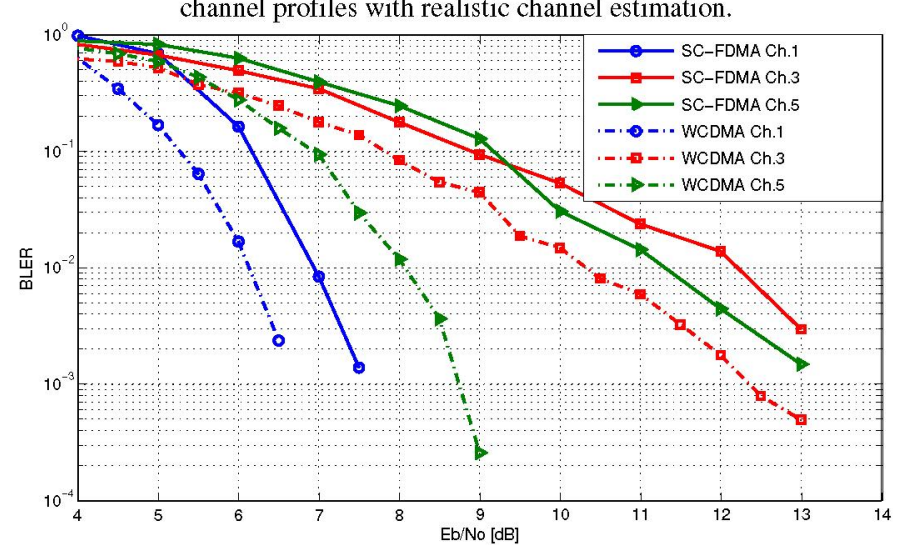

Fig. 8. Performance of WCDMA and SC-FDMA for different mobile channel profiles with TWTA (realistic channel Estimation).

Figure 8 compares the performances of the two systems in the presence of TWTA non-linear amplifier with no back off, assuming realistic channel estimation. Since, in WCDMA, only a single user is transmitting at any time, there is no superposition of the signals in the code/user domains. Thus, the signal samples have constant amplitudes; fixed at $0 \mathrm{~dB}$. Consequently, the amplifier is driven consistently at $\mathrm{IBO}=$ $O \mathrm{~dB}$ and therefore the transmitted signal is distorted only by the fixed phase shift of 47 degrees (from Figure 3). 
Concerning this in-band distortion, it is obvious from Figure 8 that this can be perfectly corrected via channel estimation. This is explained by the simulation results, as the results from Figure 8 are almost identical to those presented in Figure 7.

On the other hand, in SC-FDMA, there is a significant variation in the amplitudes of the signal samples and thus results in performance degradation compared to the results presented in Figure 7. The important point here is that the performance of the SC-FDMA system, in the presence of nonlinearity, depends substantially on the structure of the pilot sequences used for channel estimation. In particular, if the selected sequence is characterized by high PAPR in the time domain (after IFFT), then large portion of its energy will be saturated by the TWTA, resulting in poor channel estimation. So far, we have followed a non-systematic approach to design the pilot sequence, in which several pilot sequences were randomly generated in the frequency domain and the one which provided the best link performance was retained for simulation. To be more systematic, we considered CAZAC (Constant Amplitude Zero Auto-Correlation) sequence [9] as an appropriate pilot sequence for this type of scenario. Subsequently, Figure 9 shows small performance improvements are achieved with the CAZAC sequence.

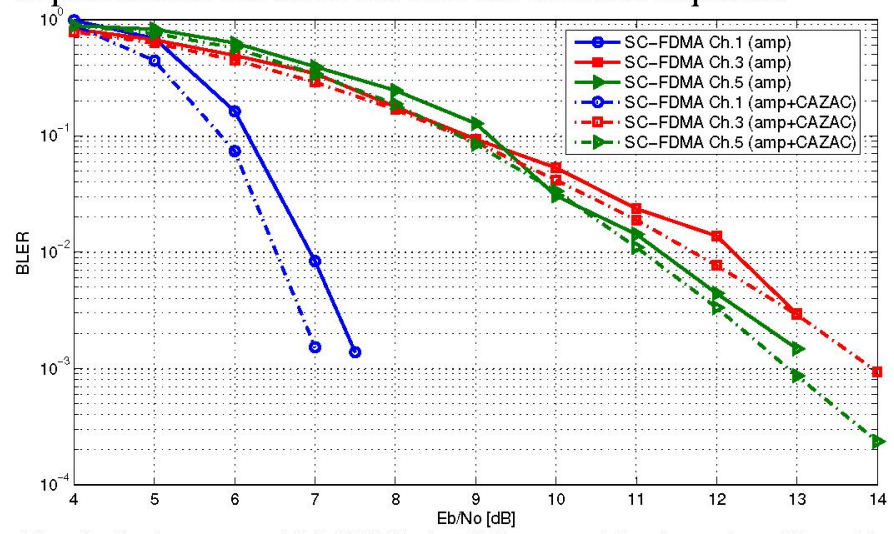

Fig. 9. Performance of SC-FDMA for different mobile channel profiles with realistic channel estimation using $\mathrm{CAZAC}$ sequence.

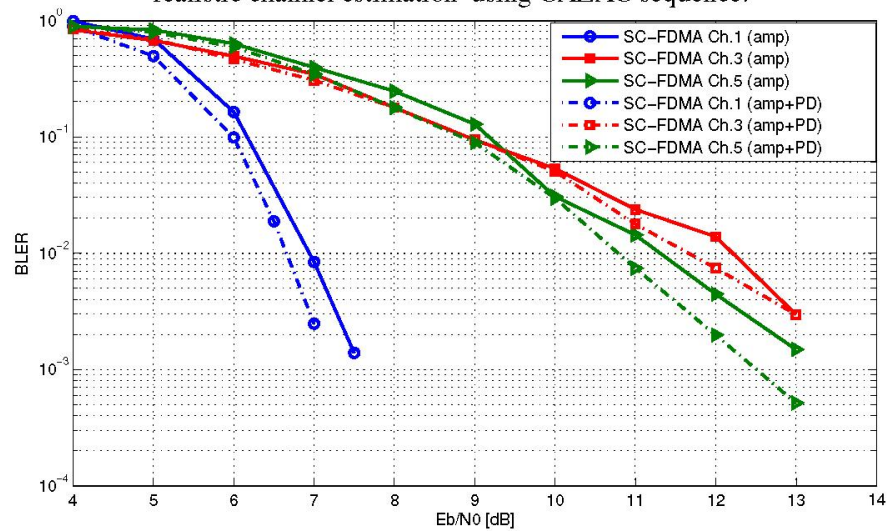

Fig, 10. Performance of SC-FDMA for different mobile channel profiles with realistic channel estimation (polynomial pre-distorter)

In addition to these results, we further extend our study to investigate the performance improvement obtained by a $2^{\text {nd }}$ order polynomial pre-distorter as a transmitter based compensation technique for the non-linear distortion introduced by the TWTA amplifier. This is demonstrated in Figure 10 with an IBO of $0 \mathrm{~dB}$ (i.e. at saturation point) is used in all cases. It is also observed that the pre-distorter gives only a small performance advantage, which is very much identical to the performance already, obtained using the CAZAC sequence. Therefore, the use of CAZAC sequence gives the same performance advantage as the polynomial pre-distorter.

\section{CONCLUSION}

In this paper, we have studied, via simulation, the performances of WCDMA (HSUPA) and SC-FDMA in the return link of an evolved-S-UMTS architecture that includes terrestrial repeaters.

We have shown that for single user transmission, both schemes provide approximately the same performance in the satellite only (case $1 \& 3$ ) environment and WCDMA shows better performance in 'satellite+ repeater' (case 5) channel environment. More non-linear effect would be expected, if the signals from many users are multiplexed in the code/user domain.

We have also shown that the in-band distortion introduced by the TWTA is perfectly compensated by channel estimation in WCDMA whereas, in SC-FDMA, 1.5-2dB degradation is observed mainly due to the non-linear amplifier. Although the polynomial pre-distorter improves the performance by about 0 $3-0.7 \mathrm{~dB}$, the same level of performance advantage is possible by adopting the CAZAC sequence for channel estimation. It can, therefore, be concluded that, as far as the link level performance is concerned, the use of the CAZAC sequence (i.e. improved channel estimation) and channel coding give similar performance to the pre-distorter. However, further investigation needs to be done on the signal spectrum in order to demonstrate its effect on the out of band radiation. In addition to this, investigation on lower channel coding rates should also be studied.

\section{REFERENCES}

[1] S. Cioni, G. E. Corazza, M. Neri, and A. Vanelli-Coralli, "OFDM vs. HSDPA comparison for Satellite Digital Multimedia Broadcasting Systems", IEEE Globecom. Conference, Dec. 2005, pp. 2922-2926.

[2] 3GPP TR 25.913 Requirements for Evolved UTRA (E-UTRA) and Evolved UTRAN (E-UTRAN, V.7.0)

[3] R. Bachl, P. Gunreben, S. Das, and S. Tatesh, "The Long Term Evolution Towards a New 3GPP* Air Interface Standard," Bell Labs Technical Journal, vol. 11, pp. 25-51, Mar. 2007.

[4] Hyung G. Myung, Junsung Lim, and David J. Goodman, "Single Carrier FDMA for Uplink Wireless Transmission," IEEE Vehicular Technology Magazine, vol. 1, no.3, pp. 30-38, Sept. 2006.

[5] H. G. Myung, J. Lim, and D. J. Goodman, "Peak-to-Average Power Ratio of Single Carrier FDMA Signals with Pulse Shaping", IEEE PIMRC'06, pp. 1-5, Sept. 2006.

[6] J. ZHANG, C. HUANG, G. LIU, and P. ZHANG, "Comparison of the Link Level Performance between OFDMA and SC-FDMA," IEEE Chinacom'06, pp. 1-6, Oct. 2006.

[7] C. Hsing-Hung, M. Ching-Shyang, W. Yeong-Cheng, and C. JiunnTsair,"Joint Polynomial and Look-Up-Table Power Amplifier Linearization Scheme," IEEE VTC'03, vol.2, pp. 1345-1349, Apr. 2003.

[8] 3GPP TR 25,808: FDD Enhanced Uplink; Physical Layer Aspects, Version 6

[9] W. Yang, H. Wei, and Z. Zhongpei, "CAZAC Sequence and its application in LTE random access," IEEE Information Theory Workshop (ITW)'06, vol. 2, pp. 544-547, Oct. 2006. 